\title{
A Follow-Up Study of the Effects of Early Malnutrition on Subsequent Development. II. Fine Motor Skills in Adolescence
}

\author{
JANINA R. GALLER, FRANK RAMSEY, AND GIORGIO SOLIMANO \\ Department of Child Psychiatry, Boston University School of Medicine, Boston, Massachusetts 02118; the \\ National Nutrition Centre, Bridgetown, Barbados, West Indies; and the Center for Population and Family \\ Health, Institute of Human Nutrition, Columbia University, New York, New York 10032
}

\begin{abstract}
One hundred nine children, aged 8 through $15 \mathrm{yr}$, who suffered from malnutrition in the 1 st $\mathrm{yr}$ of life and 107 well-nourished comparison children were tested for fine motor skills by the Purdue Pegboard test. The performance of index children was impaired on three of the four test measures. IQ measured concurrently showed a reduction in the index group; when the effects of IQ were eliminated, there was no longer any significant difference between index and comparison groups. The data suggest that the effects of early malnutrition on Pegboard performance are largely mediated by deficits in IQ. The presence of soft neurologic signs measured $4 \mathrm{yr}$ earlier in the same children was highly correlated with reduced Pegboard performance, implying that early malnutrition has effects on nervous system function that are evident at least through 15 yr of age. (Pediatr Res 19: 524-527, 1985)
\end{abstract}

\section{Abbreviations}

MANOVA, 3-way multivariate analysis of variance ANOVA, 2-way univariate analysis of variance SNS, soft neurologic signs

$R$, right-handed performance

$\mathbf{L}$, left-handed performance

$B$, both hands

A, assembly

In this report of the long-term effects of early malnutrition, we have continued to follow behavioral development in a cohort of Barbadian school children with histories of moderate to severe protein-energy malnutrition and in a matched comparison group. The earlier reports in this series described the physical and behavioral profiles of children aged 4 to 11 yr old. We now extend these observations through $15 \mathrm{yr}$ of age, at which time more than half of the children had undergone puberty.

At the younger ages, index children displayed a moderately reduced IQ and a range of deficits in behavior at school $(1,2)$. Classroom behavior was more significantly associated with school achievement than was IQ (3). A related finding was the presence of SNS in the previously malnourished children (4), as

Received May 29, 1984; accepted January 24, 1985

Reprint requests Dr. Janina R Galler. Department of Child Psychiatry, Boston University School of Medicine, 85 East Newton Street. Suite M921, Boston, MA 02118 .

This research was conducted in collaboration with the Ministries of Health and Education of Barbados and was supported by grants from the Thrasher Research Fund and the Ford Foundation. demonstrated by impaired performance on seven repetitive motor tasks first described by Denckla (5). In the present study, we have elected to apply the Purdue Pegboard test, an assessment of manual dexterity, which has been reported as being suitable for adolescents (6), and which has been successfully applied to clinic populations of this age (Rapin I, personal communication). The objective of this study was to determine whether evidence of earlier deficits as revealed by the presence of SNS would persist into adolescence.

\section{METHODS}

Site of study. This study was conducted in collaboration with the Ministry of Health at the National Nutrition Centre in Barbados. Since 1967, this Centre has provided medical care to all children with malnutrition from the time of diagnosis to 11 yr of age. All cases of malnutrition in Barbados must be reported to the National Nutrition Centre and thus, good documentation is available on a popultion of approximately 2100 malnourished children. Several features of the Barbadian population were favorable to our investigation. First, the delivery and documentation of health care are good. Almost all children are born in the Queen Elizabeth Hospital or allied facilities, and records of obstetric and perinatal care are available in almost all cases. Furthrmore, children are followed routinely by local clinics and these records were made available to us. Second, education is almost universal. Nearly all children attend school to $16 \mathrm{yr}$ of age, and $95 \%$ of the Barbadian population is literate. Comprehensive school records are available for each child. Third, the population is stable - with little in or out migration-and is largely homogeneous with respect to ethnicity and socioeconomic level which is mostly lower middle class. Because of all these advantages, we were able to obtain extensive documentation of index and comparison children during infancy, including specific details surrounding the episode of malnutrition for index children (7), during early childhood and at the time of the study.

Study population. In the earlier series of studies, 129 index children of ages 5 to 11 were selected using the following criteria.

1) They had been diagnosed on clinical evaluation by one of our group (F.R.) as showing the symptoms of protein-energy malnutrition including severe weight loss (below $75 \%$ of expected weight for age) in the absence of edema.

2) Their birth weight was at least $5 \mathrm{lb}$ (to exclude those children exposed to significant fetal growth retardation).

3) They had no evidence of prenatal or perinatal complications, as measured by standard criteria including a reduced Apgar score.

4) They had no history of convulsion, head injury, or loss of consciousness. 
The index children were hospitalized at the Queen Elizabeth Hospital for a period of $37 \pm 15$ days and were then followed by the National Nutrition Centre through 11 yr of age, where they received medical care (including home visits), nutritional counseling, and subsidized milk.

The comparison children were for the most part classmates of the index children and were matched to them by age, sex, and handedness. Except for the fact that they did not have histories of malnutrition and had normal growth during early childhood, the comparison children met the same criteria as the index children.

In the present investigation, we have followed the same child population $4 \mathrm{yr}$ later, in order to determine changes associated with growth and development into adolescence. We were successful in identifying 109 (or $85 \%$ of the original sample) of index children and 107 comparisons.

Purdue Pegboard. Testing procedures for the Purdue Pegboard have been described elsewhere in detail (8). Briefly, the instrument is a flat board containing two parallel rows of small holes into which metal pegs of about $12 \mathrm{~mm}$ are inserted. Four tasks were administered. The first was a test of $R$ in which the number of pegs inserted into holes in the right row of the Pegboard during a 30-s period was recorded. The second task was similar but tested $\mathrm{L}$. In the third test, both hands were used to insert pegs in both rows simultaneously, and the total number of pairs of pegs inserted during a 30-s interval was recorded (B). Finally, an assembly test was administered in which both hands were used to assemble a set of items, namely two washers, a peg, and a nut, in each hole of the Pegboard, and the total number of items inserted after $1 \mathrm{~min}$ were recorded (A). These procedures were applied to right-handed children only ( $90 \%$ of this population). Left-handed children were administered the left-hand task first, followed by the right-hand task. As recommended in the Purdue Pegboard Manual, the $\mathrm{L}$ of the left-handed children was considered to be in the same category for subsequent analyses as the $\mathrm{R}$ of right-handed children. All tests were administered by nurses who were blind to the background history of the child.

Data analysis. Scores on all four tasks were analyzed similarly. A MANOVA was first applied to determine whether nutritional history, the sex of the child, or his age had effects on the group of tasks. We then performed univariate analysis of variance (nutritional history $\times \operatorname{sex} \times$ age) with each individual task as the dependent variable, using three age groups of 8 to 10,11 to 13 , and 14 to $15 \mathrm{yr}$ old. ANOVA was also applied to the total score
$(\mathrm{R}+\mathrm{L}+\mathrm{B})$ since this combination is considered to be more sensitive than any of the tests alone. These analyses were supplemented by Newman-Keuls tests when appropriate.

Next, in order to examine the contribution of IQ to performance on the Purdue Pegboard, we performed a MANOVA as above, using the Full Scale IQ measured at the same time in these children (9) as a covariate. This procedure was followed by univariate analyses of covariance for the four individual tasks and the total scores $(\mathrm{R}+\mathrm{L}+\mathrm{B})$ controlling for IQ. Finally, a MANOVA was performed to determine the effects of nutritional history using SNS as a covariate.

\section{RESULTS}

Table 1 presents mean scores of the index and comparison children on the four Purdue Pegboard tasks. For the right-handed and left-handed tasks, the total number of pegs placed correctly on the board during a 30-s period was recorded, whereas the number of pairs of pegs completed were recorded for the task using both hands, and the total number of pegs and washers for the assembly task. In each instance, index children received lower scores than their comparisons, although boys and girls in index and comparison groups performed similarly.

These observations were confirmed by analysis of the data. First, we applied MANOVA (nutritional history $\times \operatorname{sex} \times$ age) with performance on all four Pegboard tasks as the dependent variable. This analysis demonstrated significant deficits for the index children contrasted with the comparisons $[F(4,201)=$ 2.85; $p<0.05]$ and significant effects of age, attributable to improved performance in the older age groups $[\mathrm{F}(8,404)=6.3$; $p<0.001]$. For this overall analysis, there were no sex effects or significant interactions.

These findings were supplemented by univariate analysis of variance (nutritional history $\times \operatorname{sex} \times$ age) for each of the Purdue Pegboard tasks individually (Table 2). As may be seen, there were significant effects of nutritional history on performance of three of the four tasks, namely the right-hand task, that performed by both hands, and the assembly task, since index children had lower scores in each instance. However, the lefthand task did not distinguish index and comparison groups. Significant age effects were present for all of the tasks, and Neuman-Keuls tests confirmed reduced performance in the 8 to 10 yr old children as compared with the two older age groups which did not differ $(\alpha=0.05)$. As was the case for the overall

Table 1. Mean values $\pm S D$ of scores achieved on the Purdue Pegboard

\begin{tabular}{|c|c|c|c|c|c|c|}
\hline \multirow[b]{2}{*}{ Task (yr) } & \multicolumn{3}{|c|}{ Males } & \multicolumn{3}{|c|}{ Females } \\
\hline & Comparison & Index & $n$ & Comparison & Index & $n$ \\
\hline \multicolumn{7}{|c|}{$1707 \quad 176$} \\
\hline $8-10$ & $12.41 \pm 0.94$ & $11.24 \pm 2.22$ & $17 / 17$ & $12.11 \pm 1.64$ & $11.83 \pm 1.76$ & $18 / 18$ \\
\hline $11-13$ & $13.53 \pm 1.72$ & $13.10 \pm 2.38$ & $32 / 30$ & $14.17 \pm 1.62$ & $13.29 \pm 2.17$ & $18 / 21$ \\
\hline $14-15$ & $12.73 \pm 2.02$ & $12.81 \pm 2.07$ & $15 / 16$ & $15.00 \pm 1.29$ & $13.00 \pm 2.16$ & $7 / 7$ \\
\hline \multicolumn{7}{|c|}{$1177+237$} \\
\hline $8-10$ & $11.47 \pm 1.50$ & $11.47 \pm 2.15$ & $17 / 17$ & $11.67 \pm 1.78$ & $11.72 \pm 2.37$ & $18 / 18$ \\
\hline $11-13$ & $13.06 \pm 1.54$ & $12.30 \pm 2.29$ & $32 / 30$ & $13.11 \pm 1.78$ & $12.43 \pm 1.69$ & $18 / 21$ \\
\hline $14-15$ & $13.20 \pm 1.74$ & $13.06 \pm 2.24$ & $15 / 16$ & $13.29 \pm 0.76$ & $12.14 \pm 2.48$ & $7 / 7$ \\
\hline \multicolumn{7}{|c|}{$061+185$} \\
\hline $8-10$ & $9.76 \pm 1.25$ & $8.94 \pm 1.43$ & $17 / 17$ & $9.67 \pm 1.33$ & $8.61 \pm 1.85$ & $18 / 18$ \\
\hline $11-13$ & $10.44 \pm 1.64$ & $10.40 \pm 2.27$ & $32 / 30$ & $11.11 \pm 1.18$ & $10.29 \pm 1.35$ & $18 / 21$ \\
\hline $14-15$ & $10.67 \pm 1.11$ & $10.63 \pm 1.75$ & $15 / 16$ & $11.29 \pm 1.50$ & $10.00 \pm 2.77$ & $7 / 7$ \\
\hline \multicolumn{7}{|l|}{ Assembly } \\
\hline $8-10$ & $23.94 \pm 4.08$ & $23.00 \pm 4.21$ & $17 / 17$ & $26.33 \pm 4.54$ & $\begin{array}{l}23.50 \pm 4.87 \\
2838+5.19\end{array}$ & $\begin{array}{l}18 / 18 \\
18 / 21\end{array}$ \\
\hline $11-13$ & $29.40 \pm 4.51$ & $26.77 \pm 6.00$ & $32 / 30$ & $30.78 \pm 5.21$ & $28.38 \pm 5.19$ & $\begin{array}{c}18 / 21 \\
7 / 7\end{array}$ \\
\hline $14-15$ & $30.27 \pm 5.66$ & $29.81 \pm 7.21$ & $15 / 16$ & $32.71 \pm 2.14$ & $27.14 \pm 7.97$ & $7 / 7$ \\
\hline
\end{tabular}


analysis, there were no sex effects on interactions by ANOVA on the individual tasks.

Finally, ANOVA was performed on the total score for tests of the right hand, left hand, and both hands $(\mathrm{R}+\mathrm{L}+\mathrm{B})$. This latter measure has been reported to provide the most sensitivity in identifying deficits in performance (8). As above, scores of index children were significantly reduced as compared with comparisons, and younger children had lower scores than did older children in this series. As above, there were no sex effects on interactions between nutritional history, sex, or age. In summary, the performance of index children was impaired regardless of the sex or age of the child.

Next, we undertook a series of analyses to test the contribution of IQ to performance on the Purdue Pegboard tasks. We have previously reported a significant reduction in IQ in previously

Table 2. $F$ values derived from ANOVA (nutritional history $\times$ sex $\times$ age) of Purdue Pegboard scores in previously malnourished children and a comparison group*

\begin{tabular}{lccc}
$\begin{array}{c}\text { Purdue } \\
\text { Pegboard } \\
\text { task }\end{array}$ & $\begin{array}{c}\text { Nutritional } \\
\text { history } \\
(\mathrm{df}= \\
1,204)\end{array}$ & $\begin{array}{c}\text { Sex } \\
(\mathrm{df}=1,204)\end{array}$ & $\begin{array}{c}\text { Age } \\
(\mathrm{df}=2,204)\end{array}$ \\
\hline $\mathrm{R}$ & $5.97 \dagger$ & 2.09 & $14.38 \ddagger$ \\
$\mathrm{L}$ & 2.62 & 0.01 & $9.8 \ddagger$ \\
$\mathrm{B}$ & $5.91 \S$ & 0.03 & $14.88 \ddagger$ \\
$\mathrm{R}+\mathrm{L}+\mathrm{B}$ & $6.97 \S$ & 0.74 & $17.52 \ddagger$ \\
$\mathrm{A}$ & $9.71 \S$ & 2.30 & $21.28 \ddagger$ \\
\hline
\end{tabular}

* There were no significant interactions between nutritional history, sex, and age.

$\dagger p<0.05$.

$\ddagger p<0.001$.

$\S p<0.01$. malnourished children through $15 \mathrm{yr}$ of age (Galler J, Ramsey F, Solimano G, unpublished data). Thus, we applied a MANOVA (nutritional history $\times$ sex $\times$ age) with the Full Scale IQ as a covariate using all four tasks as the dependent variable. Once IQ was controlled, there were no longer any significant differences between index and comparison children. However, there was a significant age effect because of lower scores among younger children $[\mathrm{F}(8,388)=8.87 ; p<0.001]$, but no significant sex effect or interactions. Similarly, univariate analyses of covariance (nutritional history $\times \operatorname{sex} \times$ age) for each individual task and also the total score $(\mathrm{R}+\mathrm{L}+\mathrm{B})$ failed to demonstrate significant differences between index and comparison groups when the effects of IQ were controlled. In summary, the reduced performance on the Purdue Pegboard by index children can in large part be attributed to deficits in IQ in these children.

Finally, we compared performance on the Purdue Pegboard with the presence or absence of SNS which had been evaluated $4 \mathrm{yr}$ earlier in this population (4). The Pearson correlation between these was $0.51(p<0.01)$ and was substantially higher for index children $(r=0.64 ; p<0.001)$ than for comparison children $(r=0.38 ; p<0.05)$. In order to test whether nutritional history effects performance on the Purdue Pegboard when scores for the SNS were controlled, we applied a 3-way analysis of covariance on the Pegboard score (nutritional history $x \operatorname{sex} x$ age) with the SNS treated as a covariate. This analysis confirmed that there were no significant differences between index and comparison children $(\mathrm{F}=1.01 ; p<0.1)$ when the contribution of SNS was eliminated. Thus, reduced Purdue Pegboard performance is correlated with impaired motor skills measured as a component of SNS.

\section{DISCUSSION}

We have previously reported that SNS, as measured by tests of motor skills developed by Denckla (5), were impaired in
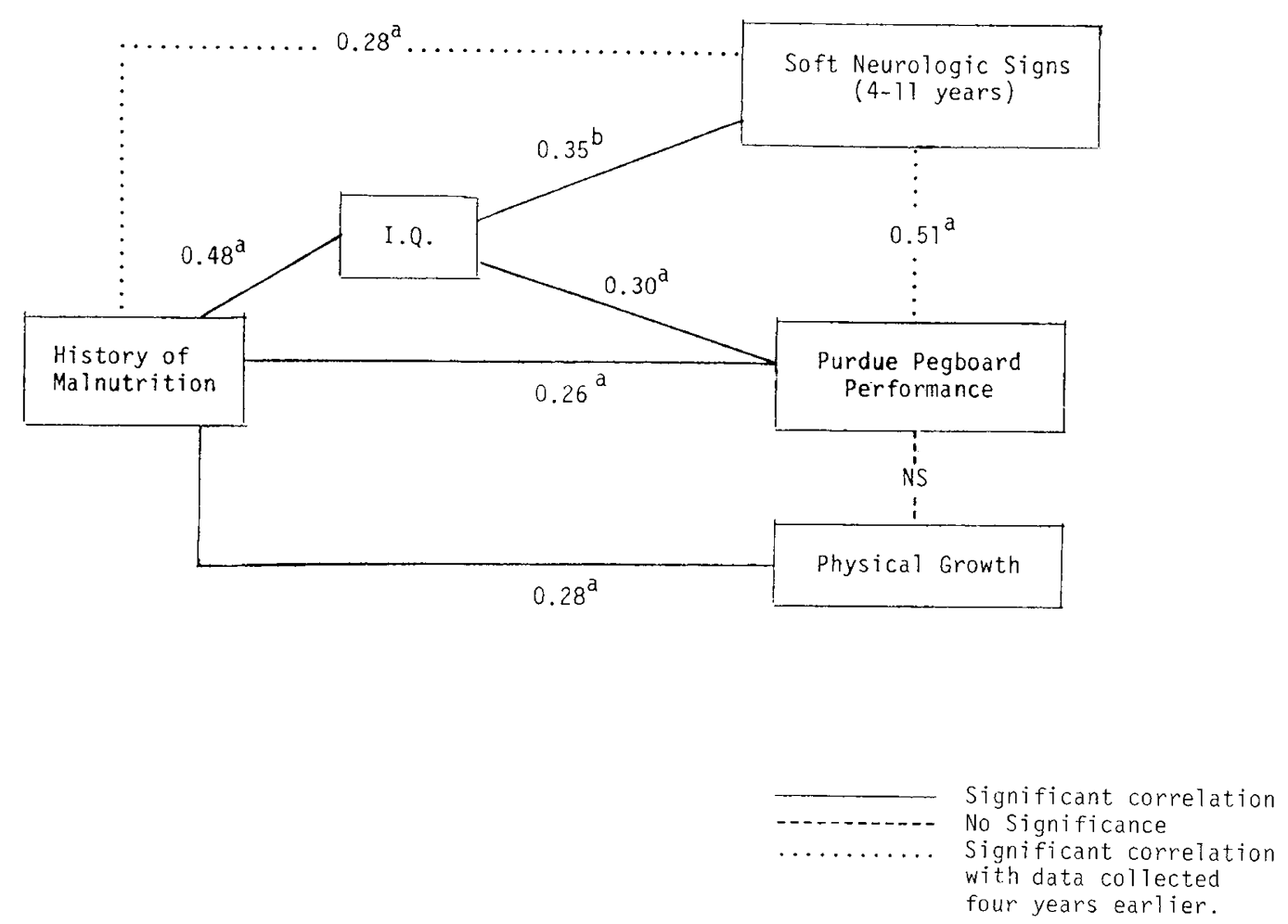

Fig. 1. A model of the interrelationships between a history of malnutrition, IQ, SNS, Purdue Pegboard performance, and physical growth at ages 8 to 11 yr. $a, p<0.001 ; b, p<0.05 ; c$, for the relationship between history of malnutrition and IQ and between history of malnutrition and Purdue Pegboard performance, a point biserial correlation was used. For the relationship between history of malnutrition and physical growth and between history of malnutrition and SNS, we used a canonical correlation. For the relationship between Purdue Pegboard performance and SNS, Purdue Pegboard performance and physical growth, and IQ and SNS we used a multiple regression. The relationship between IQ and Purdue Pegboard performance was correlated using Pearson's correlation. 
children aged 5 to $11 \mathrm{yr}$ with a history of early malnutrition (4). These tests are only appropriate for preadolescent children and in the current follow-up study of the same children 4 yr later, we have applied the Purdue Pegboard test which has been used as an appropriate challenge for older children and adults for whom norms have been established $(6,10)$.

Using this test, we have observed that children with prior malnutrition have lower scores on all tasks except those of the nondominant hand. Figure 1 shows the correlation of these scores with other functions measured at the same time and reported elsewhere, namely physical growth (11) and IQ (Galler J, Ramsey $\mathrm{F}$, Solimano $\mathrm{G}$, unpublished data), and functions tested $4 \mathrm{yr}$ earlier, namely the presence or absence of SNS (4). As may be seen in Figure 1, Purdue Pegboard performance is significantly correlated with IQ $(0.30)$, which we reported as impaired in the index group.

We found that there was a much stronger relationship between the history of malnutrition and IQ $(0.48)$ than that between prior malnutrition and Purdue Pegboard performance (0.26). When the effects of IQ were controlled, the relationship between the history of malnutrition and Purdue Pegboard performance was no longer significant. This implies that the effects of malnutrition on the Purdue Pegboard scores may be mediated by deficits in IQ. The alternate pathway, namely that malnutrition effects IQ and Purdue Pegboard performance independently, is less likely. The impact of IQ on Pegboard performance is greater in the case of performance IQ $(0.36)$ than verbal IQ $(0.25)$.

Figure 1 also records the concordant measure of physical growth (percentage of standard height and weight for age) which is impaired particularly in pubescent girls with histories of malnutrition (11). Despite the significant relationship of physical growth to a history of prior malnutrition $(0.28)$, it bore no relationship to performance on the Purdue Pegboard. It should be noted that clinical examination and biochemical tests did not suggest any evidence of concurrent malnutrition, eliminating a further cause of poor Pegboard scores.

Finally, Figure 1 expresses the relationship between SNS measured 4 yr earlier and Purdue Pegboard scores (0.51). SNS assessed the time required by index and comparison children to perform seven repetitive motor tasks, which were impaired in the index group only for the left hand and foot performance (4). The Pearson correlation between the SNS and the Purdue Pegboard was 0.51 and was even higher for index children alone $(0.64)$. Consequently, when SNS were controlled, the relationship between malnutrition and the Purdue Pegboard scores disappeared. This implies that impaired brain function is associated with early malnutrition and is expressed as reduced IQ, SNS, and impaired Purdue Pegboard scores, the three of these being closely correlated. Since SNS are most often self-correcting as age advances, the evidence here of other persistent deficits in neurologic function correlated with earlier SNS imply a more lasting defect, at least through $15 \mathrm{yr}$ of age, in the nervous system related to earlier malnutrition.

It is of interest that Purdue tests applied to the younger age group ( 8 to $11 \mathrm{yr}$ ) showed no deficit on $\mathrm{R}$, similar to the findings for SNS in the previous study, whereas for the entire group, including mostly 12 to $15 \mathrm{yr}$ olds, there was a preponderance of deficits in $\mathrm{R}$. This finding suggests a pubertal effect on perform- ance which deserves further investigation.

These findings are of significance during school years and later in life. Thus, both the test of SNS and the Purdue Pegboard test are measures of clumsiness and motor ability applied to different ages of children. Clumsiness has been correlated with the presence of learning deficits (12) and, as such, is a useful clinical tool in the neurologic evaluation of children. The motor skills tapped by these tests are also likely to be of potential importance for determining job opportunities in Barbados, including the light manufacturing and handicraft industries. To our knowledge, previous studies of the long-term consequences of malnutrition have not examined this aspect of performance.

Finally, behavioral and IQ deficits in the population are strongly correlated with the history of infantile malnutrition and only slightly correlated with socioeconomic factors and conditions of the home environment measured concurrently (9). These findings do not diminish the potential role of adverse factors in the home environment earlier in life, when the child first developed malnutrition. However, it is likely that the homogeneous conditions in Barbados and the health and nutritional care provided for these children up to 11 yr of age by the Nutrition Centre of Barbados served to reduce differences in socioeconomic and environmental conditions between index and comparison families, thereby reducing or eliminating significant interactions between early malnutrition and environmental conditions measured many years later.

Acknowledgments. The authors thank the staff of the National Nutrition Centre, Barbados for their assistance in collecting this data; Dr. Robert Harrison for statistical advice; and Dr. Isabel Rapin for recommending the use of this test.

\section{REFERENCES}

1. Galler J, Ramsey F, Solimano G, Lowell W 1983 The influence of early malnutrition on subsequent behavioral development. I) Degree of impairment in intellectual performance. J Am Acad Child Psychiatry 22:8-15

2. Galler J, Ramsey F, Solimano G, Lowell W 1983 The influence of early malnutrition on subsequent behavioral development. II) Classroom behavior. J Am Acad Child Psychiatry 22:16-22

3. Galler J, Ramsey F, Solimano G 1984 The influence of early malnutrition on subsequent behavioral development. III) Learning disabilities. Pediatr Res 18:309-313

4. Galler J, Ramsey F, Solimano G, Kucharski L, Harrison R 1984 The influence of early malnutrition on subsequent behavioral development. IV) Soft neurologic signs. Pediatr Res 18:826-832

5. Denckla MD 1974 Development of motor coordination in normal children. Dev Med Child Neurol 16:729-741

6. Seigel M, Hirschorn B 1958 Adolescent norms for the Purdue pegboard tests. Personnel Guidance J 36:363-365

7. Ramsey F 1980 Protein-Energy Malnutrition in Barbados. Josiah Macy, Jr. Foundation, New York, NY

8. Tiffon J 1968 Purdue Pegboard Manual. Science Research Assoc. Inc., Chicago, IL

9. Galler J, Ramsey F, Solimano G, Kucharski LT 1985 The influence of early malnutrition on subsequent behavioral development. VI) The role of the microenvironment of the household. Nutr Behavior (in press)

10. Tiffon J, Asher EJ 1948 The Purdue pegboard: norms and studies of reliability and validity. J Appl Psychol 32:234-247

11. Galler J, Ramsey F, Solimano G 1985 A follow-up study of the effects of early malnutrition on subsequent development. I. Physical growth and sexual maturation during adolescence. Pediatr Res 19:518-523

12. Reuben RN. Bakwin H 1968 Developmental clumsiness. Pediatr Clin North Am 15:601-610 